\title{
Development of Modified Incompressible Ideal Gas Model for Natural Draft Cooling Tower Flow Simulation
}

\author{
Tomáš Hyhlík ${ }^{1, \star}$ \\ ${ }^{1}$ CTU in Prague, FME, Department of Fluid Dynamics and Thermodynamics, Technická 4, 16607 Prague, Czechia
}

\begin{abstract}
The article deals with the development of incompressible ideal gas like model, which can be used as a part of mathematical model describing natural draft wet-cooling tower flow, heat and mass transfer. It is shown, based on the results of a complex mathematical model of natural draft wet-cooling tower flow, that behaviour of pressure, temperature and density is very similar to the case of hydrostatics of moist air, where heat and mass transfer in the fill zone must be taken into account. The behaviour inside the cooling tower is documented using density, pressure and temperature distributions. The proposed equation for the density is based on the same idea like the incompressible ideal gas model, which is only dependent on temperature, specific humidity and in this case on elevation. It is shown that normalized density difference of the density based on proposed model and density based on the nonsimplified model is in the order of $10^{-4}$. The classical incompressible ideal gas model, Boussinesq model and generalised Boussinesq model are also tested. These models show deviation in percentages.
\end{abstract}

\section{Introduction}

The working principle of natural draft wet-cooling tower is connected with releasing of latent heat during water evaporation. This effect is connected with a decrease of moist air density. There is a density difference between surrounding and inside of cooling tower which can create a natural draft. The density change in the natural draft cooling tower is not related only to heat and mass transfer between water and moist air, but there is also the change due to gravity.

The aim of this article is to identify the influences affecting the density change inside the natural draft wetcooling tower. There are several simplified density models like Boussinesq model or so called incompressible ideal gas model. The article deals with the testing and modifying of known simplified density models using data from the non-simplified model of flow, heat and mass transfer.

This work is motivated by an effort to get numerical method which provides results quickly. The numerical solutions based on previously published model [1] of flow, heat and mass transfer with general equation of state is very slow. Due to the fact, that the value of velocity in the cooling tower is in the order of meters per second, it seems natural that changes in pressure, temperature and density are almost independent of velocity except for the fill zone. The flow in the natural draft wet-cooling tower is one of the problems where Mach mumber $M a \rightarrow 0$.

\section{Governing equations of quasi one-dimensional model}

System of governing equations for quasi one-dimensional flow is

$$
\frac{\partial(\mathbf{W} A)}{\partial t}+\frac{\partial(\mathbf{F} A)}{\partial z}=\mathbf{Q}
$$

where vector of conservative properties $\mathbf{W}$ and vector of fluxes $\mathbf{F}$ are

$$
\mathbf{W}=\left[\begin{array}{c}
\rho \\
\rho v \\
\rho w_{v} \\
\rho e
\end{array}\right], \quad \mathbf{F}=\left[\begin{array}{c}
\rho v \\
\rho v^{2}+p \\
\rho v w_{v} \\
v(\rho e+p)
\end{array}\right] .
$$

Vector of sources is

$$
\mathbf{Q}=\left[\begin{array}{c}
A(z) \sigma_{v}(z) \\
p \frac{\mathrm{d} A}{\mathrm{~d} z}-A(z)\left(\rho g+\zeta \rho \frac{v^{2}}{2}\right) \\
A(z) \sigma_{v}(z) \\
A(z)\left(\sigma_{\text {qmodel }}(z)+\rho g v\right)
\end{array}\right] .
$$

First equation is overall continuity equation, second equation is momentum equation, third equation is water vapour

\footnotetext{
^e-mail: tomas.hyhlik@fs.cvut.cz
} 


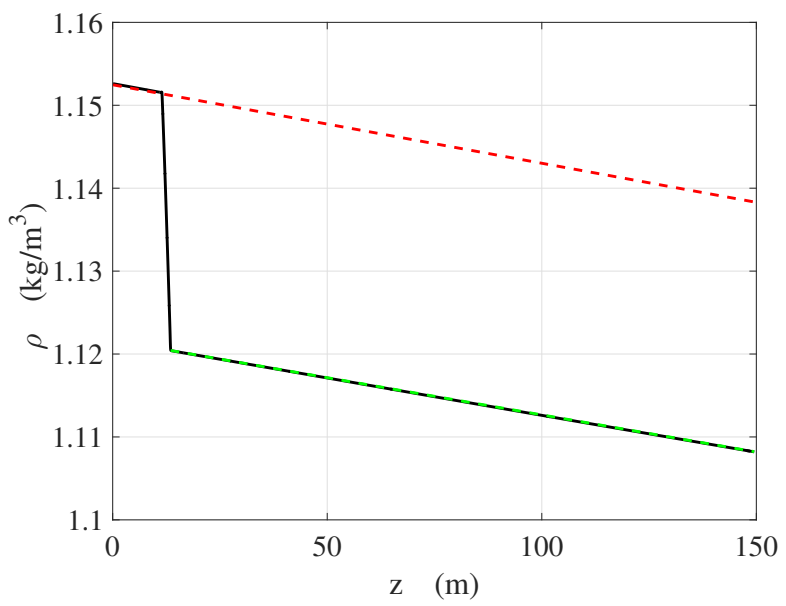

Fig. 1. Distribution of moist air density based on the numerical simulation - full black line; density distribution outside the cooling tower - dashed red line; density distribution based on hydrostatics of moist air with initial moist air parameters as calculated above the fill zone - dashed green line

continuity equation and the last equation is equation of energy. We define ad hoc internal energy of unsaturated humid air as

$$
u=\left(w_{a} c_{v_{a}}+w_{v} c_{v_{v}}\right) T=c_{v} T .
$$

Water vapour mass fraction $w_{v}$ is connected with specific humidity $x$ as

$$
w_{v}=\frac{x}{1+x}
$$

and dry air mass fraction is

$$
w_{a}=1-w_{v} .
$$

The total energy of unsaturated moist air is expressed in the form

$$
\rho e=\rho\left(c_{v} T+\frac{v_{k} v_{k}}{2}\right)
$$

Pressure can be calculated using equation of state as

$$
p=\frac{\rho R T\left(1+\frac{M_{a}}{M_{v}} x\right)}{M_{a}(1+x)}=\rho R T\left(\frac{M_{v}-w_{v}\left(M_{v}-M_{a}\right)}{M_{a} M_{v}}\right),
$$

where $M_{v}$ is molecular weight of water vapour and $M_{a}$ is molecular weight of dry air. This model is described in detail together with the method of its numerical solution in [1].

\section{Hydrostatics of moist air}

There is an equilibrium between gravity and pressure force. The pressure gradient is

$$
\frac{\mathrm{d} p}{\mathrm{~d} z}=-\rho g
$$

The moist air density at given specific humidity $x$, pressure $p$ and temperature $T$ is

$$
\rho=\frac{M_{a}(1+x)}{\left(1+\frac{M_{a}}{M_{v}} x\right)} \frac{p}{R T} .
$$

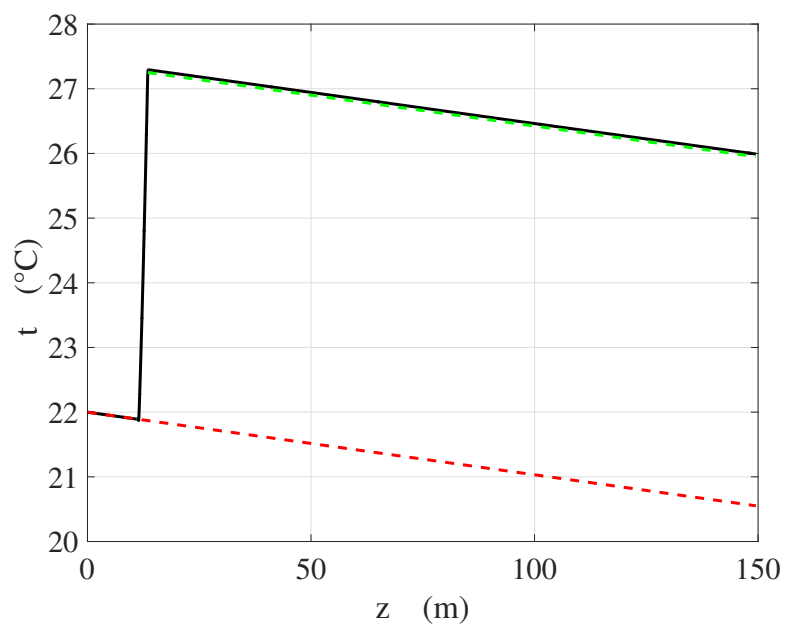

Fig. 2. Distribution of moist air temperature based on the numerical simulation - full black line; temperature distribution outside the cooling tower - dashed red line; temperature distribution based on equation (16) with initial temperature and specific humidity as calculated above the fill zone - dashed green line

After substituting equation (10) into equation (9) is

$$
\frac{\mathrm{d} p}{p}=-\frac{M_{a}(1+x)}{\left(1+\frac{M_{a}}{M_{v}} x\right)} \frac{g}{R} \frac{\mathrm{d} z}{T} .
$$

For an isoentropic proces is

$$
\frac{p}{\rho^{K}}=\text { constant }
$$

where $\kappa$ is Poisson constant of moist air

$$
\kappa=\frac{c_{p}}{c_{v}} \text {. }
$$

It is possible to substitute equation (10) into equation (12) and differentiate with respect $z$ direction similarly like in the reference [2]

$$
\frac{(1-\kappa)}{\kappa} \frac{1}{p} \frac{\mathrm{d} p}{\mathrm{~d} z}+\frac{1}{T} \frac{\mathrm{d} T}{\mathrm{~d} z}=0 .
$$

This relation is based on the assumption of constant specific humidity $x$ which is not function of $z$ direction. By using equations (9), (10) and (14) can be the temperature gradient expressed as

$$
\frac{\mathrm{d} T}{\mathrm{~d} z}=-\frac{(\kappa-1)}{\kappa} \frac{M_{a}(1+x)}{\left(1+\frac{M_{a}}{M_{v}} x\right)} \frac{g}{R} .
$$

Temperature distribution is after integration of previous equation

$$
T(z)=T\left(z_{1}\right)-\left(z-z_{1}\right) \frac{(\kappa-1)}{\kappa} \frac{M_{a}(1+x)}{\left(1+\frac{M_{a}}{M_{v}} x\right)} \frac{g}{R} .
$$

It is possible to substitute equation (16) into equation (11) and integrate with $z$ coordinate to obtain pressure distribution

$$
p(z)=p\left(z_{1}\right)\left[1-\left(z-z_{1}\right) \frac{g(\kappa-1)}{\kappa R T\left(z_{1}\right)} \frac{M_{a}(1+x)}{\left(1+\frac{M_{a}}{M_{v}} x\right)}\right]^{\frac{\kappa}{\kappa-1}} .
$$




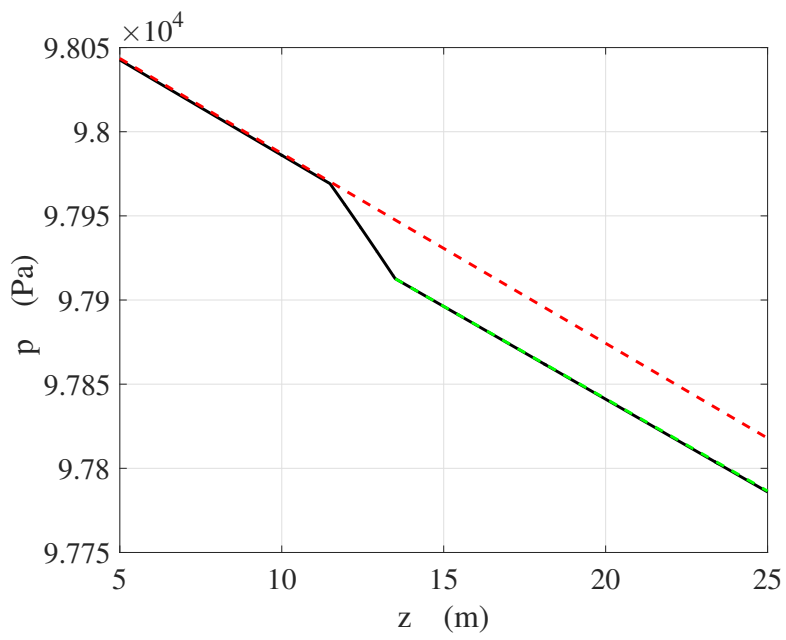

Fig. 3. Detail of the distribution of moist air pressure based on the numerical simulation - full black line; pressure distribution outside the cooling tower - dashed red line; pressure distribution based on equation (17) with initial moist air parameters as calculated above the fill zone - dashed green line

\section{Simplified density models}

One of the models used in the calculation of natural convection is Boussinesq model which is based on the definition of volumetric thermal expansion coefficient [3]

$$
\beta_{T}=-\frac{1}{\rho}\left(\frac{\partial \rho}{\partial T}\right)_{p, w_{v}} .
$$

Based on the aproximation of previous definition is

$$
\rho=\rho_{0}\left[1-\beta_{T}\left(T-T_{0}\right)\right],
$$

where $\rho_{0}$ and $T_{0}$ are constants and thermal expansion coefficiet is

$$
\beta_{T}=\frac{1}{T_{0}}
$$

It is possible to take into account change in humidity of moist air and create generalised Boussinesq model based on thermal expansion coefficient and vapour expansion coefficient

$$
\beta_{w}=-\frac{1}{\rho}\left(\frac{\partial \rho}{\partial w_{v}}\right)_{p, T} .
$$

Generalised Boussinesq model [4] is

$$
\rho=\rho_{0}\left[1-\beta_{T}\left(T-T_{0}\right)-\beta_{w}\left(w_{v}-w_{v_{0}}\right)\right],
$$

where $w_{v_{0}}$ is contant and vapour expansion coefficient is

$$
\beta_{w}=\frac{M_{a}-M_{v}}{M_{v}-w_{v}\left(M_{v}-M_{a}\right)} \approx \frac{M_{a}}{M_{v}}-1,
$$

where $M_{a}$ is molecular weight of dry air and $M_{v}$ is water vapour molecular weight.

Incompressible ideal gas model, which is taking into account humidity change is

$$
\rho=\frac{M_{a}(1+x)}{\left(1+\frac{M_{a}}{M_{v}} x\right)} \frac{p_{0}}{R T} .
$$

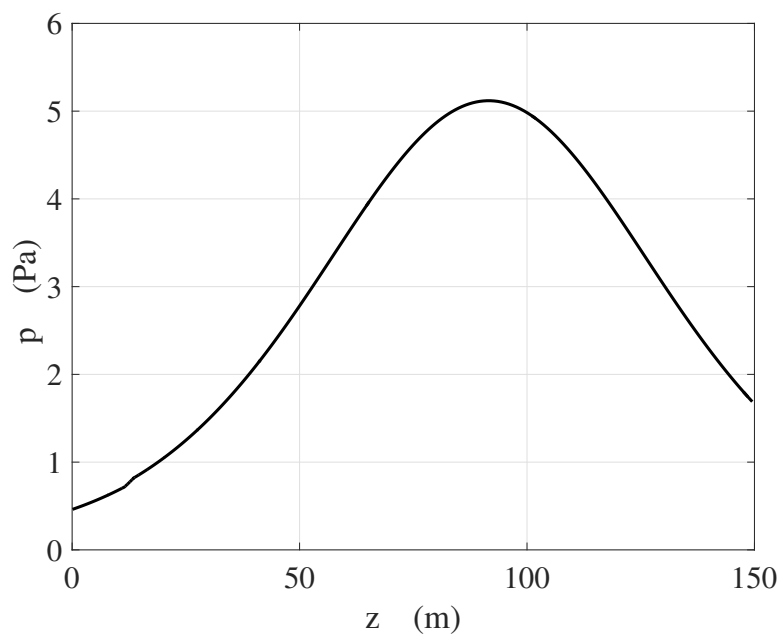

Fig. 4. Distribution of kinetic pressure based on the numerical simulation of the flow inside cooling tower

Let's take into account that dominant pressure change is connected with gravity. Incompressible ideal gas like model which is taking into account pressure change with gravity is

$$
\rho(z)=\frac{M_{a}(1+x)}{\left(1+\frac{M_{a}}{M_{v}} x\right)} \frac{p_{0}}{R T(z)}\left[1-z \frac{g(\kappa-1)}{\kappa R T_{0}} \frac{M_{a}(1+x)}{\left(1+\frac{M_{a}}{M_{v}} x\right)}\right]^{\frac{\kappa}{\kappa-1}},
$$

where $p_{0}$ is the pressure at ground level, $T$ is predicted temperature, $T_{0}$ is the temperature at ground level, $x$ is predicted specific humidity and $\kappa$ is the Poisson constant of moist air.

\section{Results}

There are comparisons of the distribution of density, temperature and pressure calculated using general model of flow, heat and mass transfer, where non-simplified equation of state is used, with distributions based on hydrostatics of moist air in figures 1,2 and 3. It is possible to observe that behaviour in the input section corresponds very well with the behaviour outside the cooling tower. It is also visible that the behaviour above the fill zone practically corresponds with the behaviour connected with hydrostatics of moist air, where the parameters above the fill are taken into account. This behaviour can be explained if figure 4 is taking into account. It is clear that kinetic pressure inside cooling tower is very low and pressure changes due to gravity field are much higher. It is possible to observe that the slope of the pressure distribution outside the cooling tower is different than the slope of pressure distribution above the fill zone which is based on the hydrostatics or on the numerical solution, but both lines intersect at the cooling tower outflow. Pressure decreased above the fill zone due to losses, temperature increased due to heat transfer, humidity increased due to mass transfer and these effects leads to the density decrease. 


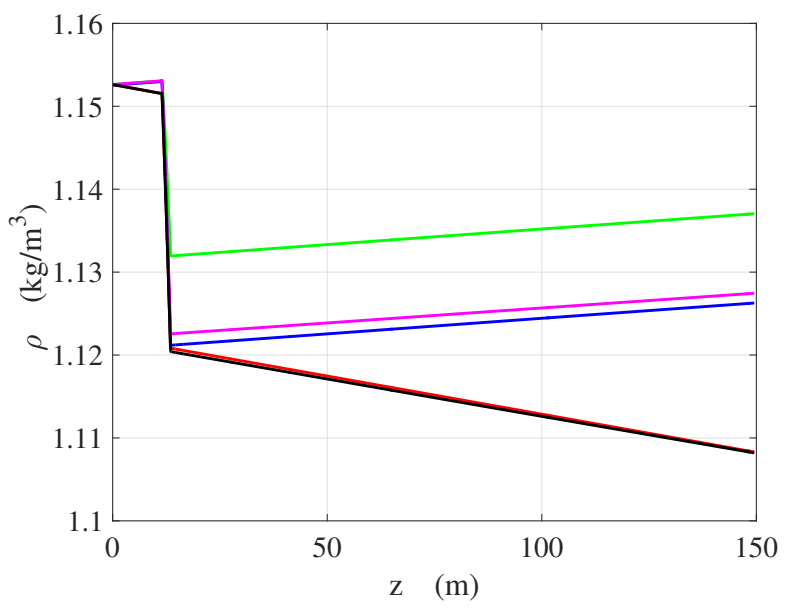

Fig. 5. Density distribution based on the numerical simulation - black line; density distribution based on modified incompressible ideal gas model represented by equation (25) - red line; density distribution based on incompressible ideal gas represented by equation (24) - magenta line; density distribution based on Boussinesq model represented by equation (19) - green line; density distribution based on generalised Boussinesq model represented by equation (22) - blue line

Figure 5 shows the comparison of the density prediction based on several simplified density models. It is depicted that the only modified incompressible ideal gas model which is taking into account hydrostatics of moist air shows a decrease above the fill similarly like the nonsimplified equation of state. It is visible that the second best model is the generalised Boussinesq model, the third best model is the classical incompressible ideal gas model and the worst is the Boussinesq model. The normalized deviations of calculated densities are shown in figure 6 . It is seen that the normalized deviations connected with modified incompressible ideal gas which is taking into account hydrostatics of moist air are in the order $10^{-4}$. The other simplified density models show normalized deviations in percentages. The biggest deviation is connected with Boussinesq model and is more than $2.5 \%$. It is possible to observe that the biggest increase of normalized deviation in the fill zone is also connected with Boussinesq model. This fact is possible to explain by ignoring changes in specific humidity in the Boussinesq model.

\section{Conclusions}

It has been shown that the behaviour of pressure, temperature and density in the natural draft wet-cooling tower corresponds very well with the behaviour of stagnant moist air in the gravity field excluding fill zone, where the heat and mass transfer occurs and minor losses have to be taken into account. Four simplified density models have been compared with the solution based on the general model. It is shown that the best match is achieved using the modified incompressible ideal gas model which is taking into account hydrostatics of moist air. Presented simplified density models are useful not only in the case of the solution of the natural draft wet-cooling towers but also in the case of the modelling of natural convection in the atmosphere. It is possible to imagine that the model which is taking into account supersaturated moist air can be created on the same basis.

Acknowledgement This work has been supported by Technology Agency of the Czech Republic under the project Advanced Technologies for Heat and Electricity Production TE01020036.

\section{References}

[1] T. Hyhlík, EPJ Web of Conferences, 92, (2015), 02027

[2] D. G. Kröger, Air-Cooled Heat Exchangers and Cooling Towers. (Penn Well Corporation, Tulsa, 2004)

[3] M.J. Moran, H.N. Shapiro, Fundamentals of engineering thermodynamics (John Wiley \& Sons, Inc., 1992)

[4] P. Sosnowski, A. Petronio, V. Armenio, International Journal of Heat and Mass Transfer, 66, (2013), 382395

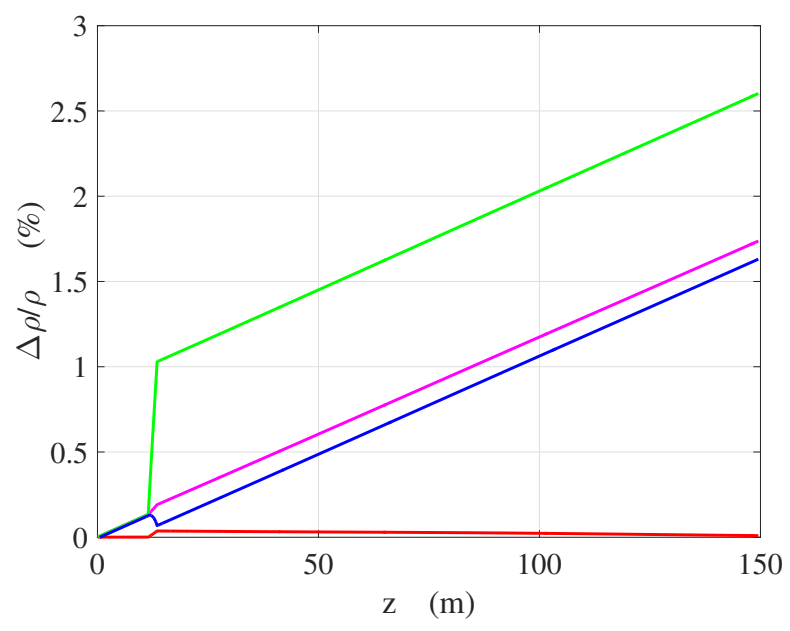

Fig. 6. Normalized deviation of density based on modified incompressible ideal gas model represented by equation (25) and density based on the numerical simulation - red line; normalized deviation of density based on incompressible ideal gas model (24) and density based on the numerical simulation - magenta line; normalized deviation of density based on Boussinesq model (19) and density based on the numerical simulation - green line; normalized deviation of density based on generalised Boussinesq model (22) and density based on the numerical simulation - blue line 